\title{
Implementación de un curso remedial para el fortalecimiento de la atención y la memoria en jóvenes con discapacidad auditiva. Inclusión en la educación superior
}

\section{Implementation of a remedial course for the strengthening of attention and memory in young people with hearing impairment. Inclusion in higher education}

\author{
MENAUT-QUIÑONES, Elsa Belem*†, ACEVEDO-MARTÍNEZ, Norma Patricia, PÉREZ- \\ GONZÁLEZ, Ivonne y GALINDO-ROSAS, Cinthia Janeth
}

Universidad Juárez del Estado de Durango. Facultad de Psicología y Terapia de la Comunicación Humana.

ID 1 ${ }^{\mathrm{er}}$ Autor: Elsa Belem, Menaut-Quiñones / ORC ID: 0000-0002-9679-1331, CVU CONACYT ID: 996075

ID $1^{\mathrm{er}}$ Coautor: Norma Patricia, Acevedo-Martínez / ORC ID: 0000-0003-4483-2389, CVU CONACYT ID: 996203

ID $2^{\text {do }}$ Coautor: Ivonne, Perez-Gonzalez / ORC ID: 0000-0002-5010-230X, Researcher ID Thomson: X-8722-2018, CVU CONACYT ID: 954229

ID $3^{\text {er }}$ Coautor: Cinthia Janeth, Galindo-Rosas / ORC ID: 0000-0001-9362-6616, CVU CONACYT ID: 997324

\section{Resumen}

Objetivo: conocer la efectividad de un curso remedial para el fortalecimiento de funciones cognitivas en jóvenes con discapacidad auditiva. Metodología: se trata de un estudio cuasi-experimental de antes y después, de corte longitudinal. Se describe la cuantificación de los puntajes naturales de las evaluaciones de funciones cognitivas mediante la Batería Neuropsicológica "NEUROPSI" Atención y Memoria, realizadas en jóvenes con discapacidad auditiva antes y después de un curso remedial previa firma de consentimiento informado. La primera evaluación fue realizada previo inicio del curso, mientras que la segunda se realizó cuando los tres jóvenes finalizaron el tercer semestre de la carrera de Terapia de la Comunicación Humana. Los datos fueron obtenidos por la revisión de los tres expedientes, de donde se tomaron las variables atención y memoria (codificación y evocación). Los resultados mostraron incremento de los puntajes en la segunda evaluación respecto a la primera evaluación en la mayoría de las subáreas de atención y memoria (codificación y evocación) evaluadas. Contribución: se identificar la importancia y pertinencia de cursos y programas enfocados a la mejora de las funciones cognitivas en jóvenes con discapacidad auditiva, los cuales brinden las herramientas que les permitan una mayor y eficiente incorporación al Sistema Educativo Superior.

Curso remedial, Funciones cognitivas, Discapacidad auditiva

\begin{abstract}
Objective: know the effectiveness of a remedial course for the strengthening of cognitive functions in young people with hearing impairment. Methodology: it's a quasi-experimental study of before and after, of longitudinal section. The quantification of the natural scores of the evaluations of cognitive functions by means of the Neuropsychological Battery "NEUROPSI" Attention and Memory, performed in young people with hearing impairment before and after a remedial course prior to signing an informed consent is described. The first evaluation was carried out prior to the start of the course, while the second was carried out when the three young people finished the third semester of the Human Communication Therapy degree. The data were obtained by reviewing the three files, from which the variables attention and memory (coding and evocation) were taken. The results showed an increase in the scores in the second evaluation with respect to the first evaluation in most of attention and memory sub-areas (coding and evocation) evaluated. Contribution: identify the importance and relevance of courses and programs focused on the improvement of cognitive functions in young people with hearing impairment, which provide the tools that allow them a greater and more efficient incorporation into the Higher Education System.
\end{abstract}

Remedial course, Cognitive functions, Hearing impairment

Citación: MENAUT-QUIÑONES, Elsa Belem, ACEVEDO-MARTÍNEZ, Norma Patricia, PÉREZ-GONZÁLEZ, Ivonne y GALINDO-ROSAS, Cinthia Janeth. Implementación de un curso remedial para el fortalecimiento de la atención y la memoria en jóvenes con discapacidad auditiva. Inclusión en la educación superior. Revista de Pedagogía Crítica. 2019, 3-9: 9-20

\footnotetext{
* Correspondencia al Autor (Correo electrónico: elsa_mvf@ @otmail.com)

$\dagger$ Investigador contribuyendo como primer autor.
} 


\section{Introducción}

Hoy en día, la inclusión de personas con discapacidad al nivel superior es una realidad en la Universidad Juárez del Estado de Durango, tal es el caso de la Facultad de Psicología y Terapia de la Comunicación Humana, en donde existen registros de alumnos con discapacidad auditiva que han logrado ingresar y terminar su carrera profesional, no obstante, en este tema aún existen muchos enigmas por resolver.

Desde los tiempos ancestrales, y aún en la actualidad, tanto en México como en el resto del mundo, la discapacidad ha hecho acto de presencia observándose como un proceso que ha ido avanzando evolutivamente para su reconocimiento y atención, pero aún con problemas que han sido señalados por el Sistema de Naciones Unidas, los cuales todavía no han sido del todo resueltos a pesar de la puesta en marcha de algunas acciones (DOF, 2014).

Actualmente, distintos sectores, entre ellos de administración pública, privada y organizaciones no gubernamentales, han mostrado gran interés por la población con discapacidad, debido a que es un grupo que requiere de especial atención, ya que esta problemática no solo afecta a la persona con discapacidad, sino también a su contexto familiar y a la comunidad a la que pertenece, con especial impacto negativo en su situación sociocultural, educativa y económica (INEGI, 2004).

Desde tiempos remotos se tenía la creencia que las personas con discapacidad auditiva tenían menos capacidades cognitivas que los normoyentes, inclusive esta creencia permanece actualmente en algunas personas (Vernon, 2005), sin embargo, los estudiantes con este tipo de discapacidad generalmente muestran un nivel de inteligencia parecido a quienes no tienen esta problemática (Federación de Enseñanza de CC. OO. de Andalucia, 2012), no obstante, en ocasiones su desarrollo cognitivo puede verse alterado por sus limitadas habilidades lingüísticas (González, 2009), ya que el lenguaje juega un papel muy importante en la actividad cognitiva (Fontal y Mejía, 2015).

\section{Justificación}

Es bien sabido que la perdida de audición influye de forma considerable en la vida de las personas que la presentan, entre ellas se puede enumerar el impacto que se refleja en su rendimiento academico (OMS, 2013), ya que una pérdida auditiva altera el proceso comunicativo de las personas con este tipo de problemática, las cuales se encuentran privadas parcial o totalmente de su input sensorial a través del órgano de la audición, y como consecuencia, presentan dificultades en la expresión oral, en las relaciones interpersonales y poseen un reducido acceso a la información de su entorno, derivando de ello necesidades educativas específicas que deben ser atendidas desde la escuela (Cañizares, 2015).

Un trastorno sensitivo, como lo puede ser una alteración o dificultad auditiva, implica privaciones en la medida en que las vias de acceso a las adquisiciones disminuyen, reduciendo los estimulos externos, asi como los centros relacionados con estos, alterando las condiciones de actividad de diversas funciones mentales (Pabón, 2009).

Por ello, en el ambito de la educación, es de suma importancia la toma de decisiones sobre aspectos que faciliten el acceso a un curriculum que muestre una adaptacion de acceso, por las dificultades que pudieran presentar las personas con discapacidad auditiva en las competencias escolares o academicas (Álvarez, 2010).

Con lo anteriormente expuesto, es posible hacer del conocimiento acerca de las necesidades educativas que presentan las personas con discapacidad auditiva, y es ahí donde radica la importancia del presente estudio, con el cual se pretende conocer la eficacia de un curso remedial implementado en jóvenes con discapacidad auditiva, el cual fue orientado al fortalecimiento de las funciones cognitivas, y de esta manera estos jóvenes aspiren a mayores posibilidades de ingreso, de permanencia y de egreso de forma más satisfactoria en estudiantes con este tipo de discapacidad, lo cual, en un futuro los ayudaran a una mejor inserción en todos los ámbitos de la sociedad, y por ende, obtendrán de esta manera la oportunidad de tener una mejor calidad de vida. 


\section{Problema}

Según la Organización Mundial de la Salud (OMS), 466 millones de personas en todo el mundo, es decir, más del 5\% de la población mundial padece pérdida de audición (OMS, 2019). Por su parte, el Instituto Nacional de Estadística y Geografía (INEGI) da a conocer los datos obtenidos a traves de la Encuesta de la Dinámica Demográfica (ENADID), donde los resultados obtenidos muestran que del total de la población con discapacidad en Mexico (7.1 millones) un $33.5 \%$ presentan dificultades para escuchar, aunque use aparato auditivo. Asimismo, la encuesta antes mencionada refiere que en el estado de Durango reside el 1.8\% de la población con discapacidad, y en lo que respecta a la discapacidad para escuchar (aunque use aparato auditivo), las personas con esta problemática corresponden al $29.9 \%$ de total de la población con discapacidad en este estado (INEGI, 2016).

Siguiendo con los datos proporcionados por la ENADID, en el año 2014 del total de la población con discapacidad de 3 a 19 años, solamente un $46.5 \%$ asistía a la escuela, observandose una diferencia de 14.1 puntos porcentuales en comparacion a la poblacion sin ninguna discapacidad (60.6\%). Lo antes expuesto solo deja ver que aún existen barreras en el ámbito educativo para la población con discapacidad, lo cual puede ser derivado por distintos factores, ya sea por la actitud de las personas, la falta de maestros bien cualificados para otorgarles educacion de acuerdo a sus necesidades, la disponibilidad y adaptabilidad de los planes de estudio, y la infraestructura de las escuelas la cual a veces no esta adaptada para individuos con alguna discapacidad.

Referente al nivel educativo se dio a conocer que de cada 100 individuos de 15 años y más con alguna discapacidad, 23 no tienen escolaridad, 45 asisten a la primaria, 15 asisten a la secundaria, 10 logran llegar al nivel medio superior y solo 7 llegan a la educación superior (INEGI, 2016). En la actualidad, aun en los países más desarrollados es poco común que los individuos con pérdida de audición lleguen a ser escolarizados, por ende, en esta población la tasa de desempleo es elevada, y quienes lograr tener un empleo lo hacen en puestos inferiores a comparación con las demás personas (OMS, 2018).
Lo anteriormente mencionado se deja ver en la poca participación económica de la población con discapacidad de 15 años y más, ya que las según la ENADID (2014), en México de cada 10 personas con esta situación, únicamente 4 participan en actividades económicas, de donde la tasa de participación económica de personas con discapacidad para escuchar (aunque use aparato auditivo) es de $35.0 \%$, y en el caso específico del estado de Durango la tasa de participación económica de 15 años y más es del $36.7 \%$. Todo parece indicar que los datos de la prevalencia de la discapacidad van a la alza, por tal motivo, es necesario desarrollar acciones que ayuden a contrarrestar las situaciones adversas a las cuales se enfrentan día con día este tipo de población (INEGI, 2016).

\section{Objetivo General}

Conocer la efectividad de un curso remedial para el fortalecimiento de funciones cognitivas en jóvenes con discapacidad auditiva.

\section{Marco Teórico}

\section{La discapacidad a lo largo de la historia}

En los tiempos de la Edad Moderna comenzó a ser estudiada por las comunidades científicas la estructura y función del ser humano, y posteriormente, en los siglos XVIII y XIX, gracias a la llegada de la Revolución Industrial se comenzó a considerar la necesidad de que el hombre fuera productivo en función del trabajo, y es a partir de ahí que aparecieron algunos cambios en respuesta a sus necesidades. Dentro de ello, comenzaron a crearse las primeras escuelas para sordomudos, así como espacios educativos para personas ciegas, donde se desarrollara un sistema universal para enseñar este tipo de personas (Hidalgo, 2018).

Así pues, para el siglo XX, las guerras de Corea y Vietnam dejaron como resultado un considerable número de personas mutiladas, ciegas, sordas y mudas; convirtiéndose en un referente de relevancia mundial, debido a que muchos jóvenes dejarían de ser productivos laboralmente, y es entonces que estas personas comenzaron a ser reconocidas como minusválidas o con trastornos mentales. 
Gracias a ello, se crearon los derechos de las personas que se encontraban es esas condiciones, expuestos en la Declaración Universal de los Derechos Humano, el Programa Internacional de Rehabilitación de Minusválidos Físicos, la Declaración de los Derechos del Retrasado Mental, la Declaración de los Derechos de los Impedidos y aprobada por la Organización Mundial de la Salud (OMS) la Clasificación Internacional de las Deficiencias Discapacidades y Minusvalías (CIDDM) (Padilla, 2010).

En México, la historia sobre las instituciones de la educación de personas con discapacidad auditiva data en Junio de 1866 con la creación de la Escuela Municipal de Sordomudos. Posteriormente, el 2 de diciembre de 1867, el presidente Benito Juárez promulgó la Ley Orgánica de Educación, donde se establece en el Capítulo II que en la Escuela para Sordomudos, los alumnos deberían aprender la lengua Española pronunciada cuando estos tuvieran aptitud para hacerlo. No obstante, la Escuela Nacional para Sordomudos tuvo resultados a finales de la década de 1960, ya que gracias a ella, muchos sordos lograron desarrollar la comunicación y el lenguaje. Tiempo después, a mediados del siglo XX algunas instituciones privadas en todo el país pretendieron brindar apoyo y atención con enfoque oralista a las personas sordas, difundiéndolo en esa época.

En el año 1953 el Instituto Mexicano de Audición y Lenguaje (IMAL) atendió a niños sordos, y a su vez formó especialistas en estas áreas. Asimismo, el Instituto Nacional de la Comunicación Humana de la Secretaría de Salud otorga atención clínica y rehabilitación a la población sorda. Por su parte, la Dirección General de la Educación Especial (DGEE), en los años 80s creo escuelas de educación especial, Grupos Integrados Específicos para Hipoacúsicos en la escuela regular, y fueron creados los primeros Centro de Rehabilitación y Educación Especial (CREE) (SEP, 2012).

En la ciudad de Durango el 25 de Marzo de 1999 se constituye la Asociación de Padres de Personas con Discapacidad Auditiva A.C. (APADAC), que tiene como objetivo brindar apoyo psicoeducativo y social a niños y jóvenes de todo el Estado con este tipo de discapacidad.
En esta institución se brinda terapia de lenguaje, terapia de aprendizaje, servicios médicos relacionados con la audición y se enseña lengua de señas a niños y jóvenes con esta problemática y público en general.

\section{Discapacidad auditiva}

La discapacidad auditiva implica una alteracion del sentido del oido, el cual es una de las vias mediante la cual el ser humano percibe el exterior, y es en función de las caracteristicas de la perdida auditiva que se puede definir el nivel de la problemática (INEGI, 2004), y es considerada sordera cuando la pérdida de audición es total, o en otro caso se considera hipoacusia, es decir, cuando existe una audicion deficiente, pero puede llegar a ser funcional con protesis o sin ellas, logrando en ocasiones adquirir el lenguaje por via auditiva, aunque en el se noten algunas deficiencias de articulación, de lexico, etc, esto dependiente del grado de la hipoacusia presentada (Martínez, 2015).

La Organización Mundial de la Salud da a conocer que un nivel de audición normal es aquel que se encuentra dentro de los 25 Decibeles ( $\mathrm{Db}$ ), se considera déficit leve cuando el nivel de audicion esta de 26 a $40 \mathrm{Db}$, déficit moderado cuando el nivel de audicion esta de 41 a $60 \mathrm{Db}$, déficit severo de 61 a $80 \mathrm{Db}$ y déficit profundo o sordera cuando el nivle de audicion se encuentra en $81 \mathrm{Db}$ o mas (OMS, 2009).

\section{Las funciones cognitivas}

Las funciones cognitivas son consideradas prerrequisitos de la inteligencia que permiten interiorizar información y facilitar el aprendizaje a través de la autoregularización del organismo, son entonces los datos acumulados en un individuo antes de adquirir un aprendizaje (Lafrancesco, 2005).

El lenguaje es de suma importancia para llegar al conocimiento del medio exterior, así como de procesos internos del mismo, (Fontal y Mejía, 2015), es decir, algunas de las funciones cognitivas se adquieren satisfactoriamente gracias a una lengua completa y accesible (Castillero y Garate, 2018). 
La atención y la memoria son funciones cognitivas indispensables para todo aprendizaje, ya que la atención permite hacer la selección de la información de relevancia y participa en la percepción del objeto de conocimiento, y la memoria almacena la información y codifica lo almacenado (Aranda y E., 2002; Seijas, 2015).

Estas funciones son de suma importancia en la vida de todo individuo, ya que son necesarios para el correcto funcionamiento de otros procesos mentales, por lo cual, es indispensable el uso de instrumentos objetivos para su evaluación (Ostrosky y otros, 2012).

\section{Funciones cognitivas en personas con discapacidad auditiva}

Se podría pensar que las personas con discapacidad auditiva al tener las mismas capacidades cognitivas que un normoyente, de igual manera su desarrollo académico debe ser similar, pero desafortunadamente no es así (Salazar, 2018), ya que en algunos casos, existen dificultades cognitivas en personas con discapacidad auditiva, esto debido a la adquisición tardía del lenguaje (Fontal y Mejía, 2015).

En lo referente al aspecto atencional Pabón (2009) menciona que la persona con discapacidad auditiva no dispone de la fuente de información a través de la audición, por ello, suele interrumpir su actividad para controlar el ambiente en forma visual, estando al pendiente de todos los estímulos de su alrededor para estar en contacto con el medio, es por ello, que a veces el estudiante con discapacidad auditiva que presenta esta conducta se le suele considerar con problemas de atención (Pabón, 2009).

Bravo (1995) menciona que después de la revisión de algunos trabajos, la mayoría de ellos revelaban que la inteligencia de las personas con pérdida auditiva puede llegar a ser tan normal como la de una persona normoyente, no obstante, en los primeros mencionados existen diferencias sobre el nivel de las habilidades ya sea en tareas de tipo verbal, así como en las tareas de tipo no verbal, ya que se debe tener muy en cuenta diferentes aspectos en ellos, tales como la interacción con los demás, el ambiente familiar, la didáctica en instituciones educativas a las que asisten o el hecho de ser hijos de padres que presentan o que no presentan discapacidad auditiva (Bravo, 1995).

\section{Inclusión educativa de personas con discapacidad}

Es bien sabido, que las personas que tienen alguna discapacidad presentan algunas desventajas en comparación con quienes no la tienen, es decir, las primeras tienen peores resultados sanitarios, resultados académicos poco satisfactorios, menor participación económica y tasas más altas de pobreza, consecuencia del limitado acceso a los servicios de salud, educación, de empleo, transporte o información que se encuentra a la disposición de esta población (OMS, 2011).

Las personas con discapacidad son un grupo minoritario, que a veces suele ser excluido de algunas áreas, entre ellas, del sistema educativo, ya que básicamente en todos los niveles, esta población no encuentra las condiciones que van de acuerdo a sus necesidades, lo cual dificulta su participación y limita el acceso a la igualdad de condiciones con sus pares que no presentan alguna discapacidad (Zárate, Díaz y Ortíz, 2017).

Hoy en día, la conceptualización sobre la discapacidad y su desarrollo en el ámbito educativo ha ido avanzado positivamente, constituyendo un camino para alcanzar la aceptación de la diversidad y de las diferencias humanas (Zapata, Bonfante, Acosta y Suárez, 2018). El mes de mayo de 2011, La Camara de Diputados del H. Congreso de la Unión, publicó en el Diario Oficial de la Federación (DOF), la Ley General para la Inclusión de las Personas con Discapacidad, donde da a conocer en el articulo $12^{\circ}$ que la Secretaría de la Educación Pública (SEP) deberá promover el derecho a la educación de las personas con alguna discapacidad, asimismo, deberá prohibir cualquier tipo de discriminación en cualquier plantel o lugar,asi como a personal perteneciente al Sistema Educativo Nacional (Cámara de Diputados del H. Congreso de la Unión, 2011).

Según la United National Educational, Scientific and Cultural Organization (UNESCO) la educación inclusiva es un proceso, el cual fortalece al sistema educativo para que logre llegar a todos los educandos, considerandolo una estrategia para alcanzar la educación para todos. 
Referente a ello, en el mes de Abril del año 2000, se realizó en Dakar el Foro Mundial sobre la Educación, donde se estableció que la educación para todos debe considerar las necesidades de grupos vulnerables, entre ellos, las personas con discapacidad o necesidades especiales de aprendizaje (UNESCO, 2009).

El objetivo de la educación inclusiva es tener una educacion de calidad, y para llegar a ello, todo sistema o centro educativo deben tratar de brindar excelencia y equidad a los alumnos (López, 2018).

En este sentido, hablando de la educación de personas con discapacidad en el nivel superior, para lograr una buena transmision de contenidos y garantizar al aprendizaje en esta población, es de vital importancia la comunicación alumno-profesor, debido a que la lengua de ambos difiere (Salazar, 2018).

\section{Evaluación Neuropsicológica "NEUROPSI" Atención y Memoria}

NEUROPSI "Atención y Memoria" es un instrumento que permite la evaluación de las funciones cognitivas, ya sea en condiciones normales o patológicas (pacientes psiquiátricos, neurológicos o con diversos problemas médicos). Dichas funciones que evalua son: Orientación, Atención (Selectiva, Sostenida y Control Atencional), Funciones Ejecutivas, y Memoria (Memoria de trabajo y Memoria a corto y largo plazo para material visoespacial y verbal).

Los reactivos por los cuales esta compuesto suelen ser sencillos y cortos, es objetivo y confiable, su aplicación es individual y ha sido utilizado en investigaciones en población hispanohablante, siendo empleado para la identificación y seguimiento en problemáticas relacionadas con atención y memoria. El tiempo de aplicación aproximado es de 50 a 60 minutos en poblacion sin alteraciones cognitivas y de 80 a 90 minutos en población con algun tipo de trastorno cognitivo.

El sistema de calificación de esta prueba neuropsicológica aporta datos tanto cualitativos, como cuantitativos (Ostrosky, 2012).

\section{Metodología}

Se trata de un estudio cuasi-experimental de antes y después, de corte longitudinal. Se describe la cuantificación de los puntajes naturales de las evaluaciones de funciones cognitivas realizadas en jóvenes con discapacidad auditiva antes y después de un curso remedial. La primera evaluación fue realizada previo inicio del curso, mientras que la segunda se realizó cuando los tres jóvenes cursaban el tercer semestre de la carrera de Terapia de la Comunicación Humana.

Los datos fueron obtenidos por la revisión de los tres expedientes.

Criterios de inclusión para el presente estudio:

Expedientes de jóvenes con discapacidad auditiva que tomaron el curso remedial y posteriormente ingresaron a la Licenciatura de Terapia de la Comunicación Humana.

Criterios de exclusión:

- Expedientes donde los participantes mostraran alteraciones psiquiátricas, psicológicas, etc.

Criterios de eliminación:

- Expedientes incompletos que no contaran con toda la información requerida.

\section{Instrumento de evaluación}

Para la evaluación de las funciones cognitivas en jóvenes con discapacidad auditiva se empleó la Batería Neuropsicológica "NEUROPSI" Atención y Memoria, de donde al analizar los expedientes se tomaron las variables de atención y memoria.

En cuanto a la variable atención fueron tomados mediante la consulta de los expedientes los resultados específicos de las subescalas de: retención de dígitos en progresión, cubos en progresión, detección visual aciertos, detección de dígitos total y series sucesivas. 
Asimismo, mediante la consulta de los expedientes, de la variable Memoria (Codificación) se tomaron los resultados de: curva de memoria volumen promedio, pares asociados volumen promedio, memoria lógica promedio historias, figura del Rey-Osterreith y codificación de caras; y para el apartado de Memoria (Evocación) se tomaron los resultados de memoria verbal espontánea total, memoria verbal por claves total, memoria verbal reconocimiento total, pares asociados total, memoria lógica promedio historias, figura del Rey-Osterreith y reconocimiento de caras total.

\section{Participantes}

Fueron analizados para la presente investigación los expedientes de 3 jóvenes con discapacidad auditiva, quienes formaban parte de la Asociación APADAC, y posteriormente ingresaron a la Facultad de Psicología y Terapia de la Comunicación Humana (FAPyTCH) de la Universidad Juárez del Estado de Durango; a quienes se les evaluó funciones cognitivas antes del curso remedial y después (tercer semestre de la carrera) mediante la Batería Neuropsicológica "NEUROPSI" Atención y Memoria.

Mediante la información de la historia clínica, se tiene como antecedentes escolares de los jóvenes que tomaron el curso remedial que había quienes en su infancia cursaron la educación primaria en un Centro de Atención Múltiple (CAM), y otros que asistían a escuelas de educación particular. Posteriormente, todos ingresaron a la educación secundaria, permaneciendo por tres años, para después continuar con su preparación en la educación media superior, contando siempre con el apoyo e intervención de intérpretes durante las clases y el desarrollo de trabajos y tareas extra-clase, hasta que finalmente culminaron este nivel educativo. Cabe mencionar que estos jóvenes siempre contaron con apoyo educativo también por parte de APADAC.

\section{Procedimiento}

El proceso para la realización del curso remedial para jóvenes con discapacidad auditiva se llevó a cabo de la siguiente manera: inicialmente, la presidenta de la Asociación de Padres de Personas con Discapacidad Auditiva de Durango A.C.
(APADAC) solicitó a la Universidad Juárez del Estado de Durango (UJED) la inclusión de jóvenes con discapacidad auditiva a algunas de las Licenciaturas de esta universidad, a lo cual, la Institución Educativa Superior accedió, proponiendo con ello un curso remedial para estos jóvenes previo ingreso a las licenciaturas.

Posteriormente, se realizó la invitación formal para la inclusión a la universidad a los jóvenes con discapacidad auditiva pertenecientes a APADAC, que previamente ya habían cursado la educación básica y la educación media superior, y que estuvieran interesados en continuar con la educación superior.

Al ser realizada la invitación por parte de la UJED para la inclusión de jóvenes con discapacidad auditiva al nivel superior, 8 de ellos refirieron interés por ingresar a la universidad, mismos a quienes se les propuso tomar el curso remedial y participar en el presente proyecto de investigación.

Después de ello, se procedió a la firma del consentimiento informado por parte de los padres y de los jóvenes con discapacidad auditiva que decidieron tomar el curso remedial, para enseguida realizar la respectiva historia clínica con la ayuda de sus padres.

Así pues, en Junio del año 2009 fue aplicada a los participantes la Batería Neuropsicológica "NEUROPSI" Atención y Memoria, esto con la finalidad de conocer las habilidades cognitivas que los jóvenes participantes tenían previo al ingreso a la universidad.

La evaluación fue realizada de manera individual y su aplicación fue por parte de un psicólogo con el apoyo de un intérprete de lengua de señas. Al dar por terminadas las evaluaciones se analizaron los resultados obtenidos y se identificaron las necesidades cognoscitivas de los participantes. Así pues, teniendo en cuenta las necesidades cognoscitivas de los jóvenes con discapacidad auditiva se diseñó y se puso en marcha por parte de la Facultad de Psicología y Terapia de la Comunicación Humana el curso remedial, teniendo como objetivo el fortalecimiento de las funciones cognitivas en estos jóvenes. 
El curso remedial antes mencionado fue diseñado e instrumentado por un Médico en Comunicación Humana, perteneciente al cuerpo académico de la FAPyTCH, quien con el apoyo de un equipo multidisciplinario, integrado por una Terapeuta de la Comunicación Humana, un maestro de Educación Especial, un Lingüista, una Psicóloga, un maestro en Lengua de Señas, un maestro en Informática y un Intérprete de lengua de Señas, lograron llevar a cabo el programa.

El curso remedial tuvo inicio en el mes de Julio del año 2009 y finalizó en el mes de Julio del 2010. Al término del curso remedial únicamente tres de los ocho jóvenes refirieron interés por cursar la Licenciatura de Terapia de la Comunicación Humana, e ingresaron al curso propedéutico que ofreció la carrera en el mes de agosto del 2010. Posteriormente, al finalizar estos jóvenes el curso propedéutico, ingresaron a la carrera en el mes de Febrero del 2011.

Con la finalidad de darle seguimiento al curso remedial, estos jóvenes contaron con el apoyo de intérpretes de lengua de señas quienes los apoyaban en el proceso enseñanzaaprendizaje dentro del aula, y por las tardes, recibían apoyo por parte de asesores pedagógicos quienes les ayudaban en el repaso de contenidos escolares, monitoreo de tareas, aclaración de dudas, preparación de exámenes y apoyo en el trabajo de investigación interdisciplinario final de cada semestre.

Al término del tercer semestre de la licenciatura en Terapia de la Comunicación Humana les fue aplicada nuevamente la Batería Neuropsicológica "NEUROPSI" Atención y Memoria, para enseguida ser analizados los resultados obtenidos de esta segunda evaluación.

Para el análisis de los datos se cuantificaron las puntuaciones naturales de cada subprueba de las áreas evaluadas.

\section{Resultados}

Los datos de la presente investigación fueron obtenidos mediante la revisión de 3 expedientes de jóvenes con discapacidad auditiva, de los cuales 2 son del sexo femenino y 1 del sexo masculino. La edad de los participantes al momento de la primera evaluación se encontró en un rango de 18 a 20 años.
Ahora bien, en cuanto a los resultados en el área de atención del sujeto 1, las subpruebas que registraron un incremento de la puntuación en la segunda evaluación con respecto a la primera fueron: cubos en progresión, detección visual de aciertos, detección de dígitos total y series sucesivas; observándose un mayor incremento en la subprueba de detección visual aciertos (Ver Grafica 1).

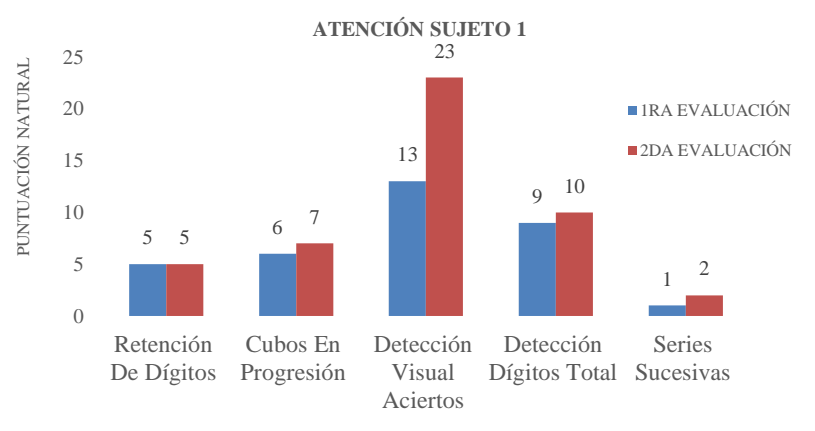

Gráfica 1 Resultados de la primera y segunda evaluación del área de la atención del sujeto 1

En el área de memoria (codificación) del sujeto 1 , se puede observar un incremento en las subpruebas de curva de memoria volumen promedio y figura de Rey-Osterreith, esta última mostrando un mayor incremento de la puntuación en la segunda evaluación respecto a la primera evaluación (Ver Gráfica 2).

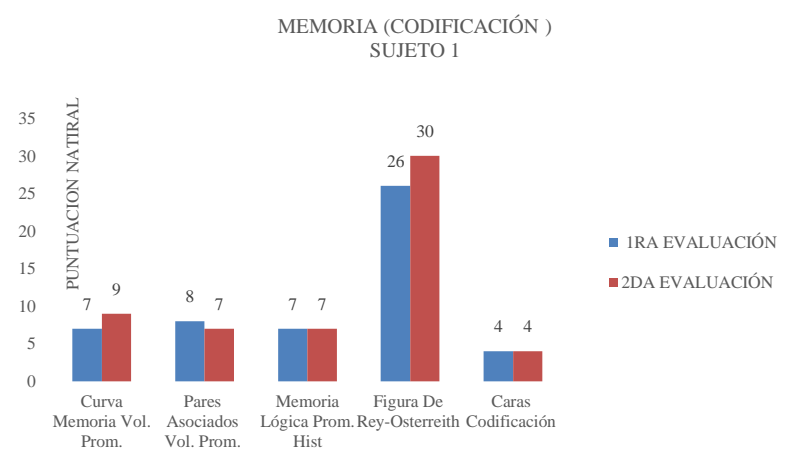

Gráfica 2 Resultados de la primera y segunda evaluación del área de memoria (codificación) del sujeto 1

En el proceso de evocación (Memoria) en el sujeto 1, se obtuvo incremento en las subpruebas de memoria verbal espontánea total, memoria verbal claves total, memoria verbal reconocimiento total, memoria lógica promedio historias y figura de Rey-Osterreith, mostrándose un mayor incremento en la puntuación en esta última (Ver Gráfica 3). 


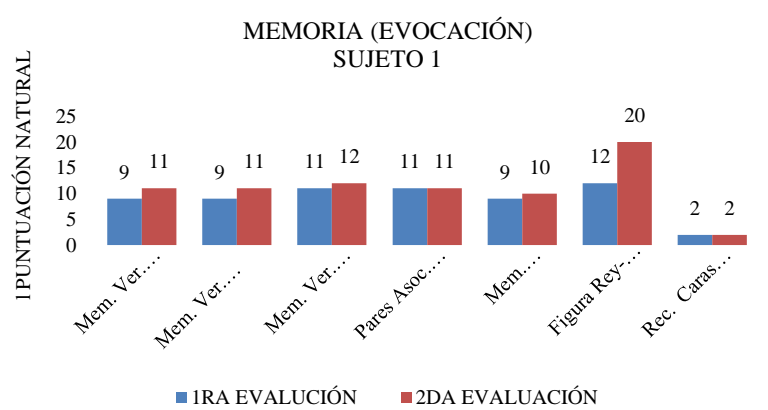

Gráfica 3 Resultados de la primera y segunda evaluación del área de memoria (evocación) del sujeto 1

En lo que respecta a la atención en el sujeto 2, las subpruebas donde se muestra incremento en el puntaje son: retención de dígitos en progresión, cubos en progresión, detección visual aciertos y series sucesivas, siendo esta última al igual que detección visual aciertos, las subpruebas donde se observó mayor incremento de la puntuación en la segunda evaluación respecto la primera. (Ver Gráfica 4).

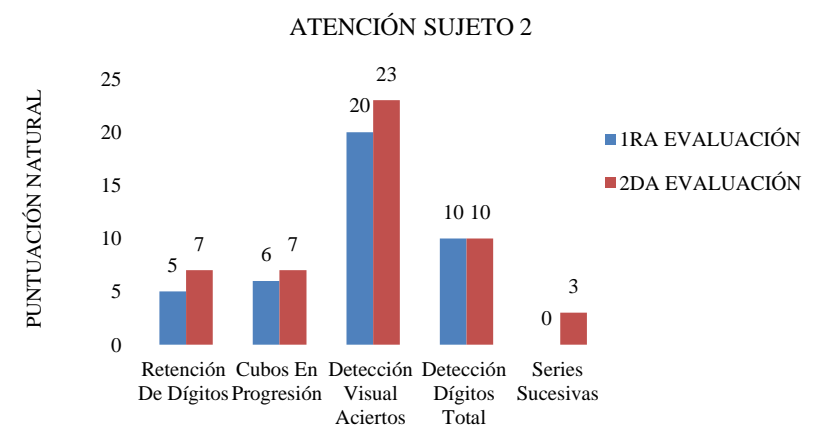

Gráfica 4 Resultados de la primera y segunda evaluación del área de la atención del sujeto 2

En el área de memoria (codificación), en el sujeto 2 se observa que las subpruebas donde se dio un incremento en el puntaje de la segunda evaluación respecto a la primera son: curva de memoria volumen promedio, pares asociados volumen promedio, memoria lógica promedio historias y figura de Rey-Osterreith, observándose mayor incremento del puntaje en esta última (Ver Gráfica 5).

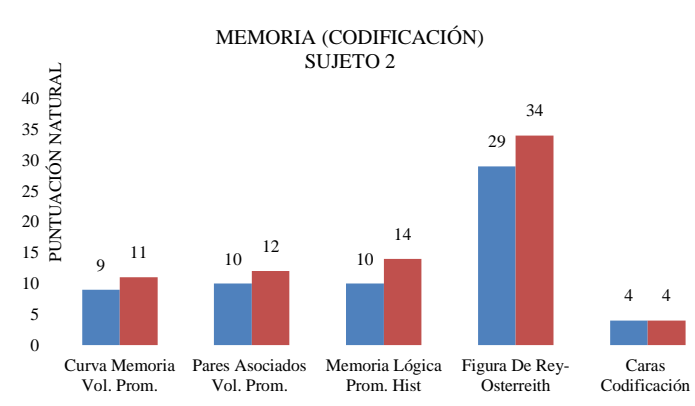

Gráfica 5 Resultados de la primera y segunda evaluación del área de memoria (codificación) del sujeto 2
En cuanto al proceso de evocación (memoria), en el sujeto 2, las subpruebas donde se incrementó el puntaje son: memoria verbal espontánea total, memoria verbal claves total, memoria verbal reconocimiento total, pares asociados total, memoria lógica promedio historias y figura de Rey-Osterreith, observándose un mayor incremento en la subprueba de figura Rey-Osterreith (Ver Gráfica $6)$.

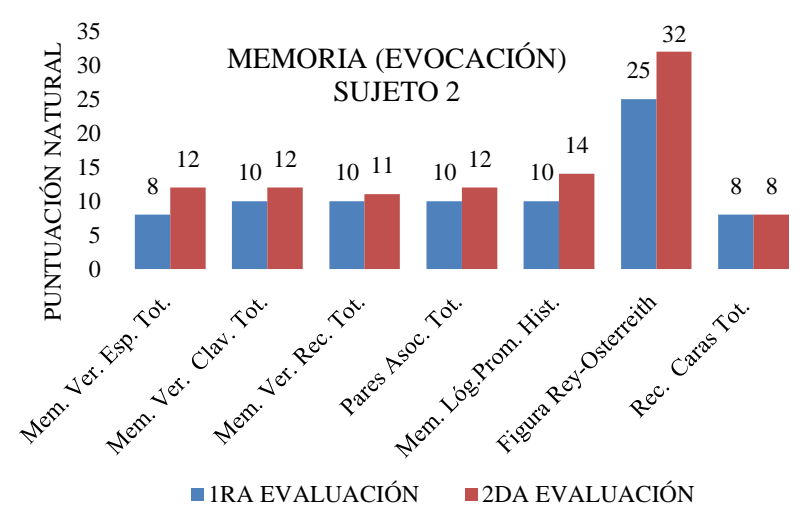

Gráfica 6 Resultados de la primera y segunda evaluación del área de memoria (evocación) del participante 2

Ahora bien, en cuanto al área de atención, en el sujeto 3 se obtuvo un incremento en todas las subpruebas, observándose un mayor incremento en la subprueba detección visual aciertos en la segunda evaluación respecto a la primera (Ver Gráfica 7).

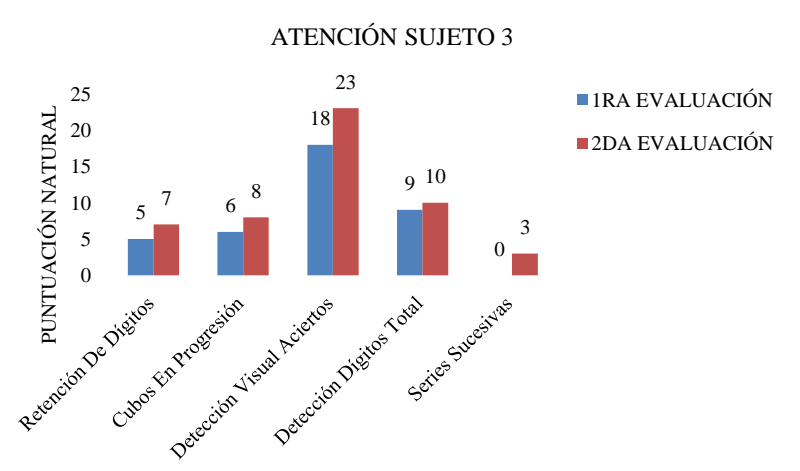

Gráfica 7 Resultados de la primera y segunda evaluación del área de la atención del participante 3

Asimismo, en el área de memoria (codificación), las subpruebas que mostraron incremento en la segunda evaluación respecto a la primera en el sujeto 3 fueron: curva de memoria volumen promedio, memoria lógica promedio historias y figura de Rey-Osterreith, observándose un mayor incremento en estas dos últimas subpruebas (Ver Gráfica 8).

MENAUT-QUIÑONES, Elsa Belem, ACEVEDO-MARTÍNEZ, Norma Patricia, PÉREZ-GONZÁLEZ, Ivonne y GALINDO-ROSAS, Cinthia Janeth. Implementación de un curso remedial para el fortalecimiento de la atención y la memoria en jóvenes con discapacidad auditiva. Inclusión en la educación superior. Revista de Pedagogía Crítica. 2019 


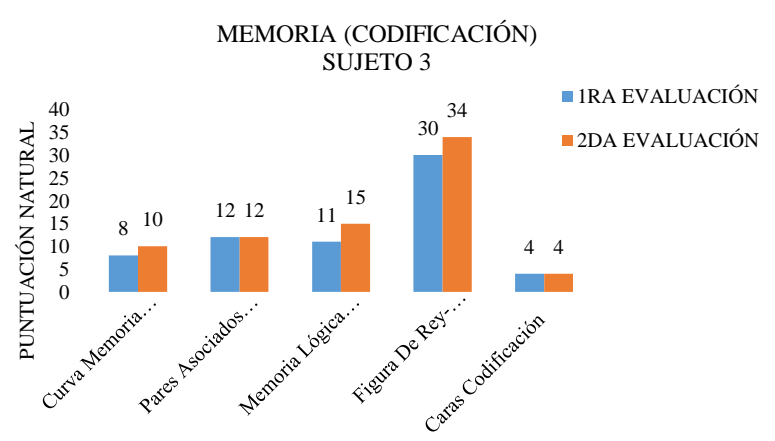

Gráfica 8 Resultados de la primera y segunda evaluación del área de memoria (codificación) del sujeto 3

En el proceso de memoria (evocación) el sujeto 3, obtuvo incremento de puntaje en la segunda evaluación respecto a la primera en las siguientes subpruebas: memoria verbal espontánea total, memoria verbal claves total, memoria verbal reconocimiento total, pares asociados total, memoria lógica promedio historias y figura de Rey-Osterreith, en esta última observándose un mayor incremento de puntaje (Ver Gráfica 9).

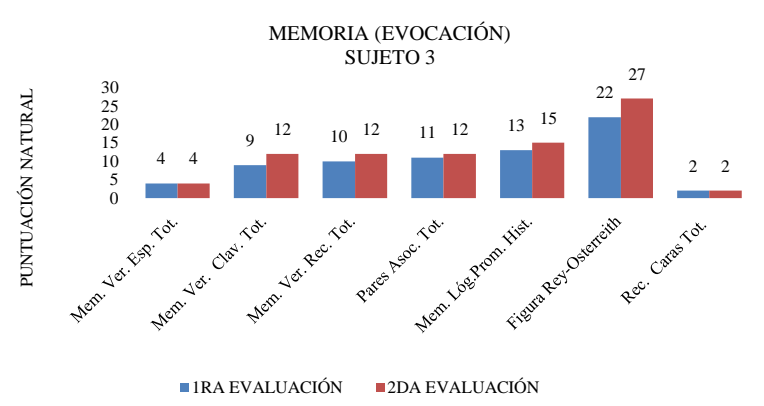

Gráfica 9 Resultados de la primera y segunda evaluación del área de memoria (evocación) del participante 3

\section{Conclusiones}

Los resultados obtenidos a través de la presente investigación, permitieron observar un mejor desempeño en los jóvenes con discapacidad auditiva en las áreas de atención y de memoria (codificación y evocación) después de haber recibido el curso remedial. Referente a ello Martinez Flores et. al. (2016) mencionan que hoy en día, a pesar de que en el tema de la educación inclusiva ya se está trabajando en las universidades de México, aún falta camino por recorrer, sin dejar de considerar que hablar de inclusión no solo es lograr el ingreso de personas con discapacidad al nivel superior, sino del apoyo constante al alumno con el cual se garantice su permanencia, un aprendizaje satisfactorio y la culminación en tiempo y forma de este tipo de estudiantes (Martinez, Delgado, Moreno y Hernández, 2016).
Actualmente, resulta un tanto controversial hablar sobre el modelo educativo más apropiado para lograr "la inclusión" de las personas con discapacidad auditiva, especialmente en la educación superior (Gomez, 2013), ya que incorporar a individuos con alguna discapacidad al sistema educativo implicaría hacer cambios tanto en el sistema, como en las propias escuelas, en donde el éxito de estos centros educativos inclusivos se logra en gran medida en función al compromiso de la legislación apropiada del país, la clara orientación normativa y la adopción de planteamientos más centrados en el alumno, lo cual conllevaría cambios en los planes de estudio, métodos y materiales de enseñanza entre otros; en resumidas palabras, al incremento de la financiación, y de esta manera, lograr que los alumnos con discapacidad puedan tener el mismo nivel educativo como quienes no tienen esta condición (OMS, 2011).

Gomez (2013) menciona que en Europa y Latinoamérica hacia finales de los años 90s se comenzó a tener el conocimiento de personas con discapacidad auditiva que culminaban sus estudios de educación básica y secundaria, y posteriormente ingresaban al nivel superior, donde se obtuvieron avances significativos a favor de la diversidad; y en el caso de México han existido casos de integración en algunos estados del país, entre ellos, Durango. No obstante, es desconocido el trabajo y las adecuaciones implementadas debido a la falta de sistematización de las propuestas y experiencias que vivieron las personas con discapacidad auditiva al interior de la universidad (Gomez, 2013). En la presente investigación los resultados obtenidos permiten identificar la importancia y pertinencia de cursos y programas enfocados a la mejora de las funciones cognitivas en jóvenes con discapacidad auditiva, los cuales brinden las herramientas que les permitan una mayor y eficiente incorporación al Sistema Educativo Superior.

\section{Agradecimiento}

Un sincero agradecimiento a la Facultad de Psicología y Terapia de la Comunicación Humana de la Universidad Juárez del Estado de Durango por las facilidades brindadas para que se llevara a cabo la investigación, e igualmente a la Asociación de Padres de Personas con Discapacidad Auditiva A.C. (APADAC). 


\section{Referencias}

Álvarez Alcazar, J. (2010). Intervención educativa en alumnos con discapacidad auditiva. Revista Digital Para Profesionales de la Enseñanza, 1-8.

Aranda, R., \& E., R. (2002). Educación especial: áreas curriculares para alumnos con necesidades educativas especiales. España: PEARSON EDUCACION. S.A.

Bravo, C. M. (1995). Desarrollo cognitivo y problemas escolares en sordos/as. Revista Pedagógica(10), 213-222.

Cámara de Diputados del H. Congreso de la Unión. (2011). Ley General Para la Inclusión de Personas con Discapacidad. México: Diario Oficial de la Federación.

Cañizares, G. (2015). Alumnos con déficit auditivo. Un nuevo método de enseñanzaaprendizaje. España : NARCEA, S.A. DE EDICIONES.

Castillero Obaldía, A., \& Garate-Estes, M. (2018). Maximizando el potencial de los niños, jóvenes y adultos sordos. Panamá : FUGA Editorial .

DOF. (2014). Programa Nacional para el Desarrollo y la Inclusión de las Personas con Discapacidad 2014-2018.

Federación de Enseñanza de CC. OO. de Andalucia. (2012). Deficiencia auditiva. Revista Digital para Profesionales de la Enseñanza, 18 .

Fontal González, A., \& Mejía Zuluaga, C. (2015). Construcción del conocimiento de las personas sordas. Una aproximación a sus características sociofamiliares. Informes Psicológicos, 47-66. doi:http://dx.doi.org/10.18566/infpsicv15n2a03

Gomez Tovar, R. M. (2013). La Inclusión de la Persona Sorda a la Educación Superior. Revista Latinoamericana de Educación Inclusiva, 8(1), 93-108.

González Castellano, A. M. (2009). La discapacidad auditiva en educación. Revista Digital para Profesionales de la Enseñanza, 17.
Hidalgo Sanchez, K. (2018). Caminos NASA para pensar, sentir y vivir con condiciones físicobiológicas, psicológicas y espirituales especiales. Universidad del Valle, 1-79.

INEGI. (2004). Las personas con discapacidad en México. Una visión censal. México: INEGI .

INEGI. (2016). La discapacidad en México, datos al 2014. . México : Instituto Nacional de Estadística y Geografía .

Lafrancesco V, G. M. (2005). La evaluación integral y del aprendizaje. Fundamentos y estrategias. Bogotá : COOPERATIVA EDITORIAL MAGISTERIO.

López-Velez, A. L. (2018). La escuela inclusiva. El derecho a la equidad y la excelencia educativa. Servicio Editorial de la Universidad del Pais Vasco.

Martinez Flores, F. G., Delgado Sanchez, U., Moreno Aguirre, A. J., \& Hernández Bucio, L. S. (2016). Inclusión educativa de personas sordas señantes en nivel universitario en México. ConCiencia, 1(2), 43-56.

Martínez Pérez, H. F. (2015). Comunicación, desempeño laboral y discapacidad auditiva. Revista Científica de Ciencias Humanas(32), 23-43.

OMS. (2009). Cuidado primario del oido y la audicion. Material de capacitación. Geneva: Organización Mundial de la Salud.

OMS. (2011). RESUMEN. Informe Mundial Sobre la Discapacidad. . Malta : Organización Mundial de la Salud.

OMS. (2013). Millions of people in the world have hearing loss that can be treated or prevented. Inís Communication .

OMS. (2018). Sordera y pérdida de la audición. OMS .

OMS. (2019). Sordera y pérdida de la audición. Organización Mundial de la Salud .

Ostrosky, F., Gómez, M. E., Matute, E., Roselli, M., Ardila, A., \& Pineda, D. (2012). NEUROPSI. Atención y Memoria. 2da Edición. El Manual Moderno, 1-62.

MENAUT-QUIÑONES, Elsa Belem, ACEVEDO-MARTÍNEZ, Norma Patricia, PÉREZ-GONZÁLEZ, Ivonne y GALINDO-ROSAS, Cinthia Janeth. Implementación de un curso remedial para el fortalecimiento de la atención y la memoria en jóvenes con discapacidad auditiva. Inclusión en la educación superior. Revista de Pedagogía Crítica. 2019 
Pabón Serrato, S. (2009). La discapacidad auditiva. Como es el niño sordo. Innovación y Experiencias Educativas(16), 1-10.

Padilla-Muñoz, A. (2010). Discapacidad: contexto,concepto y modelos. Revista Colombiana de Derecho Internacional(16), 381414.

Salazar Durango, M. A. (2018). Estrategias para la inclusión de estudiantes sordos en la educación superior latinoamericana. Ratio Juris, 13(26), 193-214.

Seijas Gómez, R. (2015). Atención, memoria y funciones ejecutivas en los trastornos del espectro autista. Cuanto hemos avanzado desde Leo Kanner. Rev. Asoc. Esp. Neuropsiq., 573586. doi:10.4321/S0211-57352015000300009

SEP. (2012). Orientaciones para la atención educativa de alumnos sordos que cursan la educación básica, desde el Modelo Educativo Bilingue-Bicultural. México: Secretaría de Educación Pública.

UNESCO. (2009). Directrices sobre políticas de inclusión en la educación. París: Organización de la Naciones Unidas para la Educación, la ciencia y la cultura.

Zapata Lesmes, C., Bonfante, M., AcostaSolano, J., \& Suárez, M. (2018). ResearchGate.

Zárate-Rueda, R., Díaz-Orozco, S. P., \& OrtízGuzmán, L. (2017). Educación superior inclusiva. Un reto para las prácticas pedagógicas. Revista Electrónica Educaré, 21(3), 1-24. 